\title{
Enantiospecific Generation and Trapping Reactions of Aryne Atropisomers
}

\author{
Yun-Long Wei, Guillaume Dauvergne, Jean Rodriguez,* and Yoann Coquerel*
}

ABSTRACT: Enantioenriched aryne atropisomers having a biaryl stereogenic axis vicinal to the reactive triple bond are demonstrated to exist. These reaction intermediates are easily produced in situ and can undergo the standard aryne cycloaddition chemistry in an enantiospecific manner. Notably, the aryne atropisomers herein have allowed the practical syntheses of a small nanographene as well as some triptycene and anthracene derivatives that embed stereogenic axes of controlled absolute configurations.

ortho-Arynes have a rich chemistry with plenty of applications for the synthesis of partially or fully aromatic molecules, from complex natural products to large polycyclic aromatic hydrocarbons. ${ }^{1-4}$ They are reactive electrophilic species that must be generated in situ, most often through the ortho-elimination of suitable aromatic precursors. While the synthetic chemistry of aromatic molecules is traditionally not associated with stereochemistry, chirality may result from the inherently chiral shapes of crowded polycyclic aromatic compounds as in the helicenes $^{5-7}$ or from the presence of stereogenic axes in biaryl systems $^{8,9}$ (Figure 1a). Chirality at the molecular scale is a a)

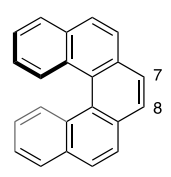

[5]helicene

b)

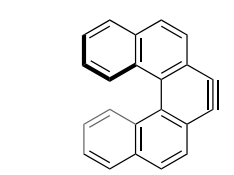

rac-7,8-[5]helicyne (1)

Tsurusaki and Kamikawa, 2017 generation \& stereoselective applications

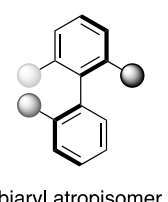

biaryl atropisomer

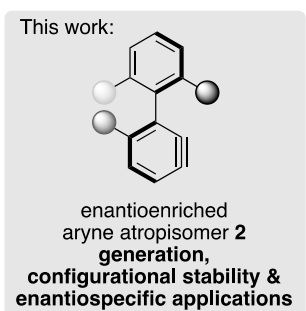

Figure 1. (a) Examples of chiral aromatic hydrocarbons; (b) some corresponding racemic and enantioenriched ortho-arynes.

property of growing importance in chemical and physical sciences, as well as in medicine and technology. ${ }^{10}$ Various additions to the electrophilic triple bond of ortho-arynes have largely been employed for the synthesis of chiral biaryls through the formation of the biaryl $\mathrm{C}\left(\mathrm{sp}^{2}\right)-\mathrm{C}\left(\mathrm{sp}^{2}\right)$ single bond, ${ }^{1,8,9,11}$ and some helicenes have also been derived from arynes. $^{12,13}$ Recently, the palladium-catalyzed cyclotrimeriza-

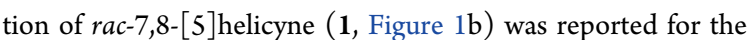
stereoselective syntheses of chiral nanographenes embedding up to six [5] helicene units. ${ }^{14-16}$ rac-7,8-[5]Helicyne (1) is an inherently chiral ortho-aryne, the first of its kind, ${ }^{17}$ and no enantioenriched inherently chiral aryne is known. Herein, we propose to extend the synthetic chemistry of arynes to chiral and enantioenriched aryne atropisomers 2 bearing a biaryl stereogenic axis vicinal to the reactive aryne triple bond (Figure 1b).

The aryne atropisomer $\mathbf{2 a}$ was selected as the molecular prototype to evaluate this idea (Scheme 1). It was devised that

Scheme 1. Retrosynthetic Analysis to Enantioenriched Aryne Atropisomers and Some Computed Properties of Aryne $2 \mathrm{a}(\mathrm{R}=\mathrm{Me})$
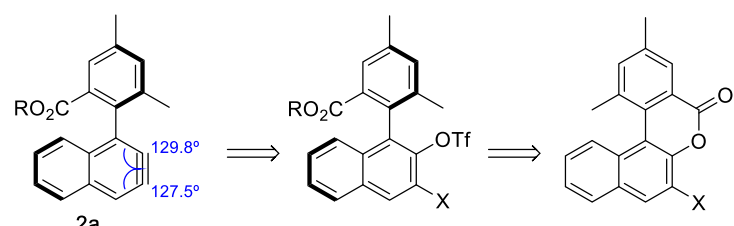

$2 a$

$\ddagger_{\text {enant }}=106.0 \mathrm{~kJ} \cdot \mathrm{mol}^{-1}$

(calculated for $\mathrm{R}=\mathrm{Me}$ )

$x=\operatorname{SiMe}_{3}, 1$

Bringmann lactones $\mathrm{X}=\mathrm{Br}$, I

enantioenriched aryne $\mathbf{2 a}$ could be generated from its so-called Kobayashi $\left(\mathrm{X}=\mathrm{SiMe}_{3}\right)^{18}$ or Suzuki $(\mathrm{X}=\mathrm{I})^{19}$ aryltriflate precursors, themselves derived from an atroposelective transesterification of some Bringmann lactones. ${ }^{20}$ The configurational stability of aryne atropisomer $2 \mathrm{a}(\mathrm{R}=\mathrm{Me})$ was evaluated computationally using DFT methods (see the 
Supporting Information for details). Its barrier to enantiomerization was calculated at $\Delta G^{\ddagger}$ enant $=106.0 \mathrm{~kJ} / \mathrm{mol}$, corresponding to a racemization half-life of $58 \mathrm{~h}$ at $25^{\circ} \mathrm{C}$, or 132 days at $0{ }^{\circ} \mathrm{C}$. The barrier to enantiomerization of the aryne atropisomer $\mathbf{2 a}$ is very significantly lower than the barrier to enantiomerization of the corresponding biaryl derivative having two $\mathrm{C}\left(\mathrm{sp}^{2}\right)-\mathrm{H}$ bonds in place of the aryne triple bond, which was computed at $\Delta G_{\text {enant }}^{\ddagger}=151.6 \mathrm{~kJ} / \mathrm{mol}$. In addition to the obvious absence of steric effect from the ortho$\mathrm{H}$ atom, the observed greater distortion toward linearity of the alkyne terminus ortho to the stereogenic axis in aryne 2a $\left(129.8^{\circ}\right.$ vs $127.5^{\circ}$, Scheme 1) may contribute to this large difference in barriers to enantiomerization. This stresses the crucial contribution to configurational stability of the ortho$\mathrm{C}\left(\mathrm{sp}^{2}\right)-\mathrm{H}$ bond in tris( ortho-substituted) chiral biaryl systems. Because the half-life of the reactive aryne triple bond in $2 \mathrm{a}$ is likely lower than its racemization half-life by several orders of magnitude, the racemization of $2 a$ itself should not be an issue under standard reaction conditions. Thus, providing that both the generation and the trapping reactions of the aryne atropisomer $2 \mathrm{a}$ are enantiospecific, some new options for the synthesis of a large array of biaryl atropisomers with controlled absolute configurations would be available.

In practice, repeating the work of Wang on the organocatalytic atroposelective opening of some Bringmann lactones $^{21}$ allowed us to obtain the bromo-naphthol 3a on a multigram scale in $82 \%$ yield and $92 \% e e$, and the original iodosubstituted analog $\mathbf{3 b}$ was prepared by the same method in $81 \%$ yield and $90 \%$ ee (Scheme 2; all molecules in schemes are

Scheme 2. Preparation of Enantioenriched Aryne Atropisomer Precursors

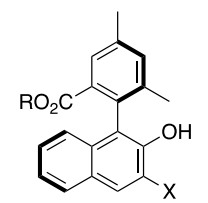

3a, $\mathrm{X}=\mathrm{Br}, 82 \%, 92 \%$ e $3 \mathbf{b}, \mathrm{X}=1,81 \%, 90 \%$ ee from the corresponding Bringmann's lactones
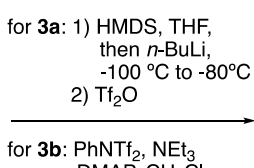

DMAP, $\mathrm{CH}_{2} \mathrm{Cl}_{2}$

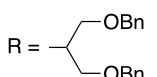

represented with their correct absolute configuration). Bromonaphthol 3a was then converted into the Kobayashi-type trimethylsilyl aryltriflate precursor 4a using the procedure developed by Pérez and Guitian, ${ }^{22}$ and the Suzuki-type iodo aryltriflate precursor $\mathbf{5 b}$ was obtained directly by sulfonylation of naphthol $\mathbf{3 b}$, both transformations occurring without detectable erosion of the enantiopurity.

The Diels-Alder cycloaddition between furan and the aryne atropisomer $\mathbf{2 a}$, derived from either $\mathbf{4 a}$ or $\mathbf{5 b}$, was selected as the benchmark reaction (Scheme 3). In an early trial, the reaction between the enantioenriched precursor 4 a $(92 \%$ ee) and excess furan in the presence of cesium fluoride afforded the expected functionalized biaryl atropisomeric cycloadduct 6a in $42 \%$ yield as a 1:1 mixture of diastereomers (Scheme $3 \mathrm{a}$ ). Remarkably, both diastereomers of 6a showed $91 \%$ ee, demonstrating that the intermediate aryne atropisomer $\mathbf{2 a}$ was indeed generated and that it retained most if not all the stereochemical information in an enantiospecific reaction, validating our initial hypothesis. However, these conditions from the Kobayashi precursor $4 \mathbf{a}$ were impeded by the
Scheme 3. Diels-Alder Cycloaddition between Furan and the Aryne Atropisomer 2a: (a) Early Experiment; (b) Optimized Conditions

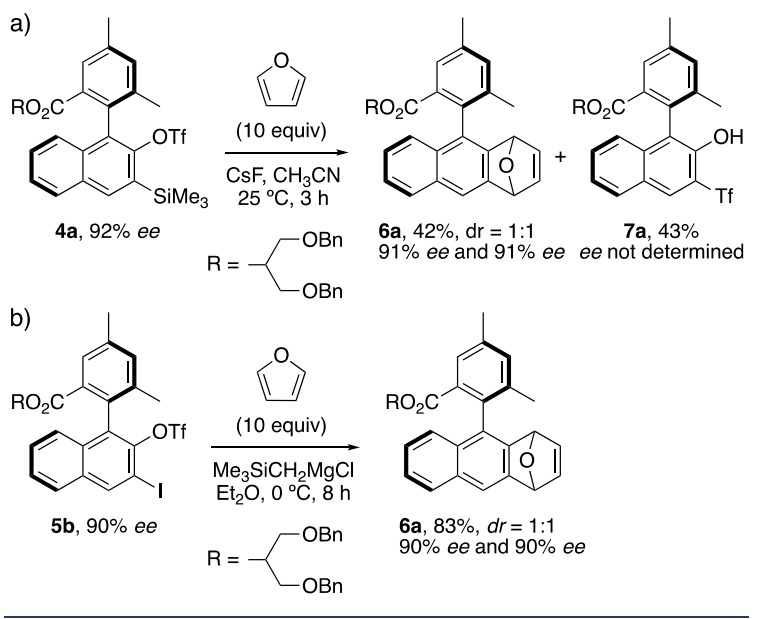

migration of the sulfonyl group onto the anionic carbon atom generated upon elimination of trimethylsilyl fluoride, leading to the formation of the thia-Fries rearrangement product $7 \mathbf{a}$ in $43 \%$ yield. Variations of the reaction conditions, as well as the use of precursors with alternative sulfonate leaving groups or functional groups, did not solve the problem (see the Supporting Information for details). From this part of the work, it was concluded not only that the enantioenriched aryne atropisomer 2a can be generated and reacted in an enantiospecific manner but also that its Kobayashi-type precursor is not suitable because of a propensity to undergo a competitive thia-Fries rearrangement instead of the expected 1,2-elimination. The formation of the undesired thia-Fries rearrangement side-product $7 \mathrm{a}$ under conditions that usually do not promote this rearrangement was attributed to steric effects from the proximal stereogenic axes in $4 \mathbf{a}$ and the corresponding anionic reactive intermediate that facilitate the $\mathrm{O} \rightarrow \mathrm{C}$ migration of the sulfonyl group for conformational reasons (see the Supporting Information for a DFT modeling study). In order to temper the thia-Fries process, the generation of the aryne atropisomer $\mathbf{2 a}$ was attempted from the Suzuki-type precursor $\mathbf{5 b}$ through ortho-magnesiated aryltriflate intermediates (Scheme $3 \mathrm{~b}$ ). ${ }^{23,24}$ After optimization (see the Supporting Information), it was ultimately found that an excess of an ethereal trimethylsilylmethyl magnesium chloride solution ${ }^{24}$ at $0{ }^{\circ} \mathrm{C}$ promoted efficient and reproducible reactions, allowing the isolation of $\mathbf{6 a}$ in $83 \%$ yield (1:1 $d r$ ) with no detectable amount of the thia-Fries product $7 \mathbf{a}$. Most importantly, both diastereomers of $\mathbf{6 a}$ were obtained with $90 \% \mathrm{ee}$ indicating virtually no erosion of enantiomeric purity from the precursor $\mathbf{5 b}$ under the optimized conditions.

In order to evaluate the enantiospecific synthetic options available with the enantioenriched aryne atropisomer $2 a$, a set of representative cycloadditions was tested, first addressing diastereoselectivity and regioselectivity issues (Scheme 4). Diels-Alder cycloadditions proved possible with two substituted furans and $\mathrm{N}$-phenylsulfonyl pyrrole affording products $\mathbf{6 b}, \mathbf{6 c}$, and $\mathbf{6 d}$ with homogeneous retention of the enantiomeric purity. In all three cases, the two possible diastereomers were obtained in a 1:1 ratio, indicating no substrate control of the diastereoselectivity from the aryne 
Scheme 4. Representative Cycloadditions with the Aryne Atropisomer 2a Focusing on Diastereoselectivity and Regioselectivity Outcomes ${ }^{a}$

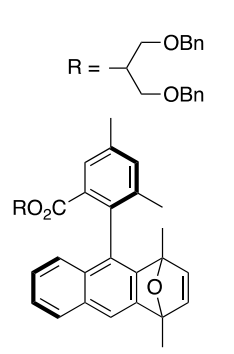

6b, $81 \%, d r=1: 1$
$90 \%$ ee and $87 \%$<smiles></smiles>

$6 c, 69 \%, d r=1: 1$
$90 \%$ ee and $85 \%$ ee<smiles>c1ccc(Cc2oc(-c3ccccc3)c3ccccc23)cc1</smiles>

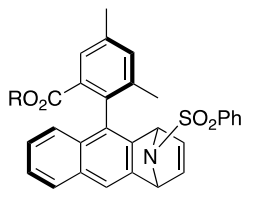

6d, $78 \%, d r=1: 1$ $0 \%$ ee and $89 \%$ ee

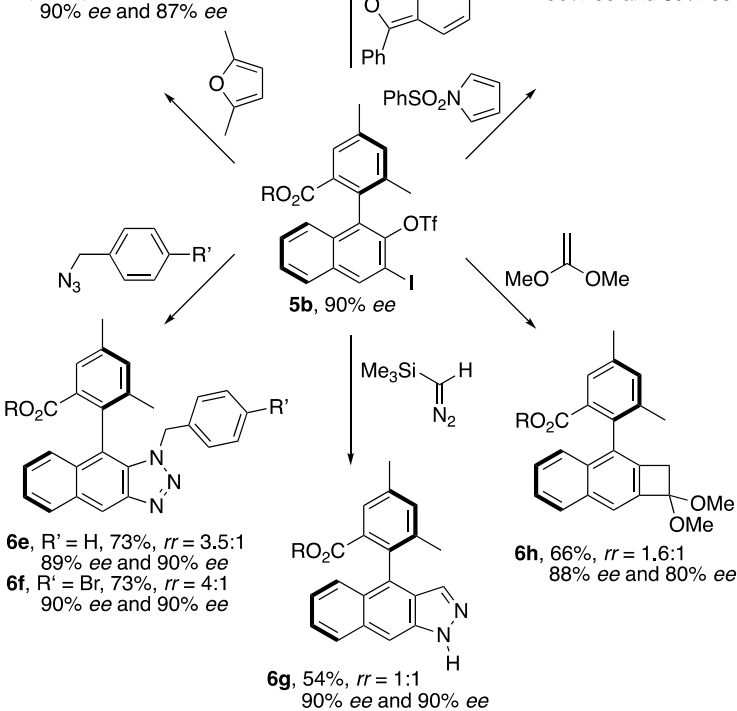

${ }^{a}$ All reactions were performed with 3-5 equiv of the arynophile and 10 equiv of $\mathrm{Me}_{3} \mathrm{SiCH}_{2} \mathrm{MgCl}$ in $\mathrm{Et}_{2} \mathrm{O}$ at $0{ }^{\circ} \mathrm{C}$. Yields were determined for isolated material. Diastereomer and regioisomer ratios $(d r$ and $r r$, respectively) derived from ${ }^{1} \mathrm{H}$ NMR analyses of the crude reaction mixtures and were confirmed on isolation, and the enantiomer ratio was determined by analytical HPLC on chiral stationary phases of the isolated material.

atropisomer 2a. Regioselectivity in the reactions of aryne atropisomer 2a was evaluated through some 1,3-dipolar cycloadditions with two azides and a diazo compound to afford cycloadducts $\mathbf{6 e}, \mathbf{6 f}$, and $\mathbf{6 g}$, and also a $[2+2]$ cycloaddition with dimethyl ketene acetal to give the benzocyclobutane $\mathbf{6 h}$. The regioselectivity in $\mathbf{6 e}$ was ascertained through the single-crystal $\mathrm{X}$-ray diffraction analysis of a derivative (see the Supporting Information, CCDC 1997613). Overall, only moderate regiocontrol was observed, but counterintuitively favoring the regioisomer involving bond formation between the nucleophilic part of the polarized reaction partner and the alkyne terminus ortho to the stereogenic axis in aryne $\mathbf{2 a}$, which is the most hindered reactive position. This selectivity is in agreement with the aryne distortion model proposed by Garg and Houk in which the nucleophile adds preferentially to the alkyne terminus with the greater distortion toward linearity (see angles in 2a, Scheme 1). ${ }^{25}$ Some small but tangible variations in the stereoretention around the stereogenic axis were observed in

the cases of the two diastereomers of $\mathbf{6 c}$ and the two regioisomers of $\mathbf{6 h}$ (Scheme 4). From the results in Schemes 3 and 4 , it seems that the generation of the aryne atropisomer $\mathbf{2 a}$, from either $\mathbf{4 a}$ or $\mathbf{5 b}$, occurs with full enantiospecificity, and it can be reasonably hypothesized that the barriers to enantiomerization in products $6 \mathrm{c}$ and $6 \mathrm{~h}$ are high enough to avoid their racemization at a significant rate under the reaction and analyses conditions. The variations in stereoretention in diastereomers $\mathbf{6 c}$ and regioisomers $6 \mathrm{~h}$ could thus be attributed to the existence of some kinetically competing cycloaddition pathways in these cases that would allow for the rotation around the stereogenic axes.

Next, the reactions of the aryne atropisomer 2a with symmetric small polycyclic aromatic hydrocarbons, as well as those of the structurally distinct aryne atropisomers derived from 5c and 5d, were assessed (Scheme 5). Notably, these reactions are free from diastereoselectivity and regioselectivity matters and proceeded with uniform retention of the stereochemical information. For instance, the annulative $\pi$ extension (APEX) ${ }^{26}$ at the bay region of perylene with aryne atropisomer 2a afforded the small chiral nanographene $\mathbf{6 i}$.

Scheme 5. Examples of Cycloadditions of Aryne Atropisomers with Small Polycyclic Aromatic Hydrocarbons ${ }^{a}$
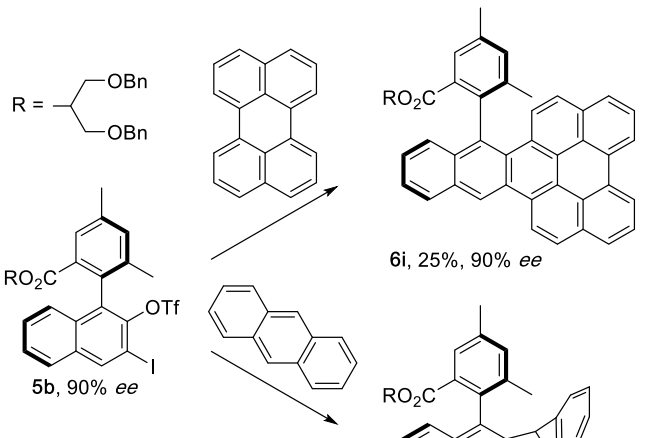

$6 \mathrm{i}, 25 \%, 90 \%$ ee
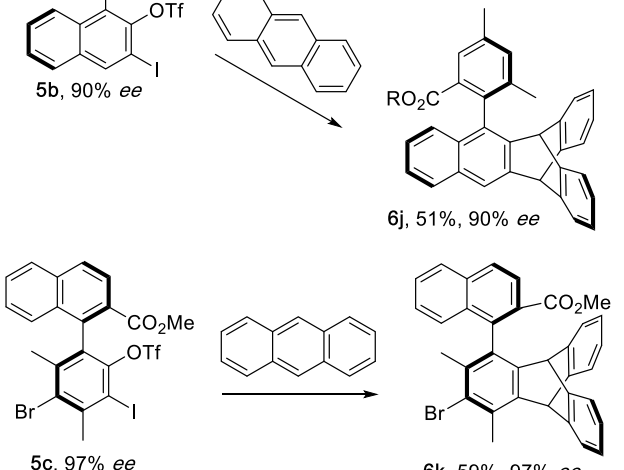

$6 \mathrm{k}, 59 \%, 97 \%$ ee
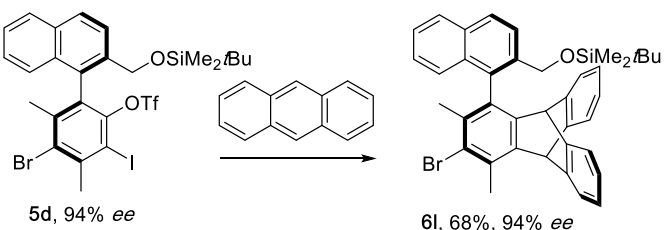

${ }^{a}$ All reactions were performed with 3-5 equiv of the arynophile and 10 equiv of $\mathrm{Me}_{3} \mathrm{SiCH}_{2} \mathrm{MgCl}$ in $\mathrm{Et}_{2} \mathrm{O}$ and/or toluene at $0{ }^{\circ} \mathrm{C}$. The enantioenriched phenol precursor of $5 \mathbf{c}$ and $5 \mathbf{d}$ (3c, not depicted) was obtained by semi-preparative HPLC on a chiral stationary phase. Yields were determined for isolated material, and the enantiomer ratio was determined by analytical HPLC on chiral stationary phases. 
Also, the $[4+2]$ cycloaddition of aryne atropisomer $2 \mathrm{a}$ with anthracene afforded the atropisomeric triptycene ${ }^{27}$ derivative $\mathbf{6 j}$, and its structural analogues $\mathbf{6 k}$ and $\mathbf{6} \mathbf{l}$ were derived from the aryne atropisomer precursors $\mathbf{5 c}$ and $\mathbf{5 d}$, respectively. The structure and absolute configuration in $\mathbf{6 k}$ were confirmed by $\mathrm{X}$-ray diffraction techniques (see the Supporting Information, CCDC 2019279). Finally, the possibility for enantiospecific deoxygenative aromatization reactions with the oxa-bridged cycloadducts $^{28}$ obtained from the reaction between the aryne atropisomer derived from $\mathbf{5 c}$ and isobenzofuran was demonstrated through the synthesis of the enantioenriched anthracene atropisomer 8 (Scheme 6).

Scheme 6. Preparation of an Enantioenriched Anthracene Atropisomer

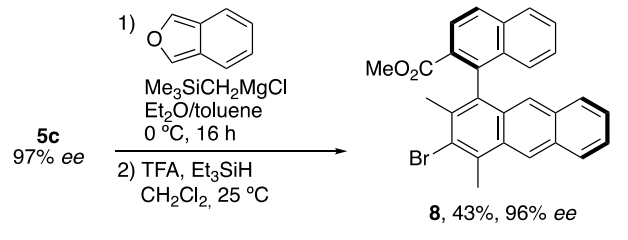

In summary, enantioenriched aryne atropisomers having a biaryl stereogenic axis vicinal to the reactive triple bond are demonstrated to exist. They can be generated in an enantiospecific manner from the corresponding ortho-iodo aryltriflates and trimethylsilylmethyl magnesium chloride, conditions mild enough to tolerate the presence of esters, some silyl ether, and bromine atoms for what was studied at this stage. Their trapping cycloaddition reactions with archetypal representative arynophiles occurred in an enantiospecific manner, unlocking new options for the stereocontrolled synthesis of nonracemic atropisomers in a broad meaning. Particularly appealing are their reactions with anthracene for the synthesis of triptycene atropisomers, and their APEX reactions with perylene and isobenzofuran, involving a complementary deoxygenative aromatization step in the latter case, for the synthesis of nonracemic large polycyclic aromatic hydrocarbon atropisomers. Studies in this direction are ongoing in our laboratory.
The authors declare no competing financial interest.

\section{ACKNOWLEDGMENTS}

Dr. Michel Giorgi (Aix-Marseille University) is gratefully acknowledged for the X-ray structural analyses of a derivative of $\mathbf{6 e}$ and 6k (CCDC 1997613 and 2019279). We are indebted to Dr. Nicolas Vanthuyne (Aix-Marseille University) for the determination of all enantiomer ratios and the resolution of $3 \mathrm{c}$ by semipreparative chiral HPLC. We thank Dr. Jean-Valère Naubron (Aix-Marseille University) for the circular dichroism spectroscopy of 3c. Dr Michel Rajzmann (Aix-Marseille University) is warmly thanked for some early calculations. Part of this work was supported by the computing facilities of the CRCMM, 'Centre Régional de Compétences en Modélisation Moléculaire de Marseille’. Financial support from Aix-Marseille University, Centrale Marseille and the CNRS is also gratefully acknowledged. Y.-L.W. thanks the China Scholarship Council for a doctoral studentship (No. 201508330296), and G.D. thanks the French government for a doctoral studentship (2018-2021).

\section{REFERENCES}

(1) Garcia- Lopez, J. A.; Greaney, M. F. Synthesis of Biaryls Using Aryne Intermediates. Chem. Soc. Rev. 2016, 45, 6766-6798 and the references therein.

(2) Diamond, O. J.; Marder, T. B. Methodology and Applications of the Hexadehydro-Diels-Alder (HDDA) Reaction. Org. Chem. Front. 2017, 4, 891-910 and the references therein.

(3) Takikawa, H.; Nishii, A.; Sakai, T.; Suzuki, K. Aryne-Based Strategy in the Total Synthesis of Naturally Occurring Polycyclic Compounds. Chem. Soc. Rev. 2018, 47, 8030-8056 and the references therein.

(4) Pozo, I.; Guitián, E.; Pérez, D.; Peña, D. Synthesis of Nanographenes, Starphenes, and Sterically Congested Polyarenes by Aryne Cyclotrimerization. Acc. Chem. Res. 2019, 52, 2472-2481 and the references therein.

(5) Rickhaus, M.; Mayor, M.; Juríček, M. Strain-induced helical chirality in polyaromatic systems. Chem. Soc. Rev. 2016, 45, 15421556.

(6) Rickhaus, M.; Mayor, M.; Juríček, M. Chirality in curved polyaromatic systems. Chem. Soc. Rev. 2017, 46, 1643-1660.

(7) Majewski, M. A.; Stępień, M. Bowls, Hoops, and Saddles: Synthetic Approaches to Curved Aromatic Molecules. Angew. Chem., Int. Ed. 2019, 58, 86-116.

(8) Wencel-Delord, J.; Panossian, A.; Leroux, F. R.; Colobert, F. Recent advances and new concepts for the synthesis of axially stereoenriched biaryls. Chem. Soc. Rev. 2015, 44, 3418-3430.

(9) Nguyen, T. T. The Use of Chiral ortho-Auxiliaries/Substituents and Remote Stereogenic Centers in Atropselective Biaryl Synthesis. Eur. J. Org. Chem. 2020, 2020, 147-155.

(10) Brandt, J. R.; Salerno, F.; Fuchter, M. J. The added value of small-molecule chirality in technological applications. Nat. Rev. Chem. 2017, 1, 45.

(11) Panossian, A.; Leroux, F. R. Lithium/Element Exchange as an Efficient Tool for Accessing Atropo- enriched Biaryls via Arynes. Chimia 2016, 70, 43-47. 
(12) Pérez, D.; Peña, D.; Guitián, E. Aryne Cycloaddition Reactions in the Synthesis of Large Polycyclic Aromatic Compounds. Eur. J. Org. Chem. 2013, 2013, 5981-6013.

(13) Wu, D.; Ge, H.; Liu, S. H.; Yin, J. Arynes in the synthesis of polycyclic aromatic hydrocarbons. RSC Adv. 2013, 3, 22727-22738.

(14) Hosokawa, T.; Takahashi, Y.; Matsushima, T.; Watanabe, S.; Kikkawa, S.; Azumaya, I.; Tsurusaki, A.; Kamikawa, K. Synthesis, Structures, and Properties of Hexapole Helicenes: Assembling Six [5] Helicene Substructures into Highly Twisted Aromatic Systems. J. Am. Chem. Soc. 2017, 139, 18512-18521.

(15) Zuzak, R.; Castro-Esteban, J.; Brandimarte, P.; Engelund, M.; Cobas, A.; Piątkowski, P.; Kolmer, M.; Pérez, D.; Guitían, E.; Szymonski, M.; Sánchez-Portal, D.; Godlewski, S.; Peña, D. Building a 22-ring nanographene by combining in-solution and on-surface syntheses. Chem. Commun. 2018, 54, 10256-10259.

(16) Yubuta, A.; Hosokawa, T.; Gon, M.; Tanaka, K.; Chujo, Y.; Tsurusaki, A.; Kamikawa, K. Enantioselective Synthesis of Triple Helicenes by Cross- Cyclotrimerization of a Helicenyl Aryne and Alkynes via Dynamic Kinetic Resolution. J. Am. Chem. Soc. 2020, 142, 10025-10033.

(17) The formation of 13,14-dibenzo[f,j]picyne through a hexadehydro-Diels-Alder (HDDA) reaction was evidenced with no mention to its chirality in: Nobusue, S.; Yamane, H.; Miyoshi, H.; Tobe, Y. [4.2] $\left(2,2^{\prime}\right)\left(2,2^{\prime}\right)$ Biphenylophanetriyne: A Twisted Biphenylophane with a Highly Distorted Diacetylene Bridge. Org. Lett. 2014, 16, 1940-1943.

(18) Himeshima, Y.; Sonoda, T.; Kobayashi, H. Fluoride-induced 1,2-elimination of $o$-trimethylsilylphenyl triflate to benzyne under mild conditions. Chem. Lett. 1983, 12, 1211-1214.

(19) Matsumoto, T.; Hosoya, T.; Katsuki, M.; Suzuki, K. New Efficient Protocol for Aryne Generation. Selective Synthesis of Differentially Protected 1,4,5-Naphthalenetriols. Tetrahedron Lett. 1991, 32, 6735-6736.

(20) Bringmann, G.; Breuning, M.; Tasler, S. The Lactone Concept: An Efficient Pathway to Axially Chiral Natural Products and Useful Reagents. Synthesis 1999, 1999, 525-558.

(21) Yu, C.; Huang, H.; Li, X.; Zhang, Y.; Wang, W. Dynamic Kinetic Resolution of Biaryl Lactones via a Chiral Bifunctional Amine Thiourea-Catalyzed Highly Atropo- enantioselective Transesterification. J. Am. Chem. Soc. 2016, 138, 6956-6959.

(22) Peña, D.; Cobas, A.; Pérez, D.; Guitián, E. An Efficient Procedure for the Synthesis of ortho-Trialkylsilylaryl Triflates: Easy Access to Precursors of Functionalized Arynes. Synthesis 2002, 14541458.

(23) Sapountzis, I.; Lin, W.; Fischer, M.; Knochel, P. Preparation of Polyfunctional Arynes via 2-Magnesiated Diaryl Sulfonates. Angew. Chem., Int. Ed. 2004, 43, 4364-4366.

(24) Yoshida, S.; Uchida, K.; Hosoya, T. Generation of Arynes Using Trimethylsilylmethyl Grignard Reagent for Activation of orthoIodoaryl or ortho-Sulfinylaryl Triflates. Chem. Lett. 2015, 44, 691-693 and the references cited therein.

(25) Medina, J. M.; Mackey, J. L.; Garg, N. K.; Houk, K. N. The Role of Aryne Distortions, Steric Effects, and Charges in Regioselectivities of Aryne Reactions. J. Am. Chem. Soc. 2014, 136, $15798-15805$.

(26) Ito, H.; Ozaki, K.; Itami, K. Annulative $\pi$-Extension (APEX): Rapid Access to Fused Arenes, Heteroarenes, and Nanographenes. Angew. Chem., Int. Ed. 2017, 56, 11144-11164.

(27) Preda, G.; Nitti, A.; Pasini, D. Chiral Triptycenes in Supramolecular and Materials Chemistry. ChemistryOpen 2020, 9, 719-727.

(28) Miyamoto, N.; Nakazawa, Y.; Nakamura, T.; Okano, K.; Sato, S.; Sun, Z.; Isobe, H.; Tokuyama, H. Synthesis of 9,10-Diarylanthracenes via $\mathrm{Mg}(\mathrm{TMP})_{2} \cdot 2 \mathrm{LiCl}-$ Mediated Benzyne Generation/[4 $+2]$ Cycloaddition and Deoxygenation of 9,10-Epoxyanthracene Intermediates. Synlett 2018, 29, 513-518. 